\title{
Invariants of germs of analytic differential equations in the complex plane
}

\author{
LEONARDO M. CÂMARA
}

Departamento de Matemática, CCE-UFES,

Av. Fernando Ferrari s/nº, Campus de Goiabeiras, 29060-900 Vitória, ES, Brasil

Manuscript received on August 13, 2004; accepted for publication on September 9, 2004; presented by MÁRCIO SOARES

\begin{abstract}
We study the classification of germs of differential equations in the complex plane giving a complete set of analytic invariants determining the analytic type of the underlying foliation. In particular we answer in affirmative a conjecture of S. Voronin, and generalize some previous results about dicritical singularities in a straightforward manner. Such problem has its origins in a conjecture proposed by R. Thom in the mid-1970s.
\end{abstract}

Key words: Singular foliations, resolution of singularities, holonomy, nonabelian cohomology.

\section{INTRODUCTION}

We study the classification of differential equations of the form $A d x+b d y$ in two variables, by their underlying foliations. As it is well known, any analytic differential equation in $\left(\mathbb{C}^{2}, 0\right)$ has a nonvoid set of integral curves through the origin, called separatrix set, and denoted by $\operatorname{Sep}(\mathcal{F})$. In Câmara 2001 we use the blow-up method in order to identify a complete set of invariants describing the analytic type of a generic subset of the above differential equations: the generalized curves. By definition a generalized curve has only a finite number of separatrices and no saddle-nodes along its minimal resolution (Seidenberg 1968). In fact, we introduce a new kind of resolution of foliations (called rectifier resolutions) taking care not only of the topological behavior of the singularities along the "solved" foliation but paying attention to the disposition of the strict transform of $\operatorname{Sep}(\mathcal{F})$, say $\operatorname{Sep}(\widetilde{\mathcal{F}})$. In fact, if $D_{j}$ is an irreducible component of the exceptional divisor of $\widetilde{\mathcal{F}}_{\text {and }} \operatorname{Sep}\left(\widetilde{\mathcal{F}}_{j}\right)$ is the restriction of $\operatorname{Sep}(\widetilde{\mathcal{F}})$ to a neighborhood of $D_{j}$, then $\operatorname{Sep}\left(\widetilde{\mathcal{F}}_{j}\right)$ will be a subset of fibers of a fibration transversal to $D_{j}$. Thence we determine the analytic type of each Hopf component (see definition below), say $\widetilde{\mathcal{F}}_{j}$, of the solved foliation by its set of singular points (up to homographic 
conjugacy class), the first jets of their singularities and the conjugacy class of the holonomy group in $\operatorname{Diff}(\mathbb{C}, 0)$. Indeed, we use classical topological methods of path lifting due to Ehersmann and analytic extension technics for nondegenerate reduced singularities (cf. Mattei and Moussu 1980, Martinet and Ramis 1983). Finally, the remaining invariants appear as characteristic classes which arise naturally from this geometric construction.

In order to extend the previous construction for any foliation, we have the following hindrances to overcome:

First consider the case of a nondicritical foliation with a Hopf component $\widetilde{\mathcal{F}}_{j}$ defined in the Hopf bundle $\mathbb{H}_{j}$ (see definition below), having just nondegenerate singularities and some saddle-nodes with strong variety (see definition below) siting over the invariant projective line $D_{j}$. Although the conjugacy class of the projective holonomy determines the analytic type of each singularity (Mattei and Moussu 1980, Martinet and Ramis 1983), we cannot guarantee that it determines the analytic type of the Hopf component of the foliation, even if $\operatorname{Sep}\left(\widetilde{\mathcal{F}}_{j}\right)$ is contained in a fibration transversal to $D_{j}$. In fact, there is no analytic extension theorem for saddle-nodes as in the nondegenerate case. As we shall see, in order to overcome this obstacle, we have to "isolate" such saddle-nodes (after blowing-up) with just one resonant saddle and then, verify that the projective holonomy of the new Hopf component determine the analytic type of the original saddle-node, by studying its Stokes phenomenon.

Now, suppose that $\widetilde{\mathcal{F}}_{j}$ has just nondegenerate singularities and at least one saddle-node with central variety (see definition below) siting on $D_{j}$. In this case the conjugacy class of the projective holonomy does not determine the analytic type of the saddle-node in general (Martinet and Ramis 1982). But exploring the "symmetries" of saddle-nodes with central variety, we can define an equivalence relation (with analytic moduli space) such that the new class of the projective holonomy determines the analytic type of $\widetilde{\mathcal{F}}_{j}$, since $\operatorname{Sep}\left(\widetilde{\mathcal{F}}_{j}\right)$ is contained in fibration transversal to $D_{j}$.

On the other hand, we recall that until now, the dicritical singularities - i.e., those with an infinite number of separatrices - are not well understood (cf. Cerveau 1999). In fact, their extended resolutions (Camacho et al. 1984) are composed by nondicritical and dicritical Hopf components (see definition below) satisfying:

(1) The nondicritical components are solved, that is, they have just reduced singularities;

(2) The dicritical components are pair-wise nonintersecting, and have no singular or tangential points in the zero section of its Hopf bundle.

We apply the same reasonings in order to obtain an extended rectifier resolution. First remark that the zero section of a Hopf bundle corresponding to a dicritical component is transversal to the foliation. It follows that the only invariants provided by it are the corners and the Chern class of its Hopf bundle (we verify this by path lifting). In particular, it follows that any two analytically equivalent dicritical foliations, namely $\mathcal{F}^{i}, i=1,2$, with dicritical components $\widetilde{\mathcal{F}}_{j, 0}^{i}$ intercepting just the nondicritical Hopf components, say $\widetilde{\mathcal{F}}_{j, k}^{i}, k=1 \cdots n_{i}$, must have the same 
global conjugacy in $\operatorname{PGL}(2, \mathbb{C})$ which does not only carry corners of $\widetilde{\mathcal{F}}_{j, 0}^{1}$ into corners of $\widetilde{\mathcal{F}}_{j, 0}^{2}$ but simultaneous conjugate all the projective holonomies of $\widetilde{\mathcal{F}}_{j, k}^{i}$ evaluated over the transversal section $D_{j, 0}^{i}$ - the zero section of the Hopf bundle determined by the dicritical component $\widetilde{\mathcal{F}}_{j, 0}^{i}$ (see figure 2). So, we cannot choose the projective holonomies of the nondicritical Hopf components freely, in the extended rectifier resolution of a germ of dicritical foliation. In other words, the cellular objects of the resolution of a dicritical singularity are no longer just the Hopf components, but the following ones:

(1) The union of a dicritical component together with the nondicritical components intercepting it, say $\widetilde{\mathcal{F}}_{j, 0} \bigcup_{k=1}^{n_{j}} \widetilde{\mathcal{F}}_{j, k}$;

(2) The nondicritical Hopf components of the foliation nonintersecting any dicritical foliation.

In fact the analytic types of these objects are determined by the projective holonomies of the nondicritical Hopf components of the foliation in the extended rectifier resolution and by the tree of corners and singularities of each cell. It follows that this objects are suitable for the definition of a good covering for the first cohomology set of the isotropy group of a foliation model as in Câmara 2001. This work was presented in the International Conference Geometry and Foliations 2003 held at Ryukoku University, Kyoto, Japan.

\section{BASIC DEFINITIONS AND NOTATIONS}

A germ of singular foliation, say $(\mathcal{F}: \omega=0)$, in $\left(\mathbb{C}^{2}, 0\right)$ of codimension 1 is, roughly, the set of integral curves of a given germ of 1 -form $\omega \in \Omega^{1}\left(\mathbb{C}^{2}, 0\right)$ which may be assumed to have just an isolated singularity at the origin. Let $\operatorname{Diff}\left(\mathbb{C}^{k}, 0\right)$ (respect. $\left.\widehat{\operatorname{Diff}}\left(\mathbb{C}^{k}, 0\right)\right)$ be the group of germs of analytic (respect. formal) diffeomorphisms of $\left(\mathbb{C}^{k}, 0\right)$ fixing the origin and $\operatorname{Diff}_{\mathbf{I}}\left(\mathbb{C}^{k}, 0\right) \subset$ $\operatorname{Diff}\left(\mathbb{C}^{k}, 0\right)$ (respect. $\left.\widehat{\operatorname{Diff}}_{\mathbf{I}}\left(\mathbb{C}^{k}, 0\right) \subset \widehat{\operatorname{Diff}}\left(\mathbb{C}^{k}, 0\right)\right)$ be the subset of such diffeomorphisms, tangent to the identity. We say that two germs of foliations in $\left(\mathbb{C}^{2}, 0\right)$, namely $\left(\mathcal{F}_{j}: \omega_{j}=0\right), j=1,2$, are analytically conjugate if there is $\Phi \in \operatorname{Diff}\left(\mathbb{C}^{2}, 0\right)$, such that $\Phi$ sends leaves of $\mathcal{F}_{1}$ into leaves of $\mathcal{F}_{2}$. We say that $h_{1}, h_{2} \in \operatorname{Diff}(\mathbb{C}, 0)$ are analytically conjugate if there is $\phi \in \operatorname{Diff}(\mathbb{C}, 0)$ such that $\operatorname{Ad}\left(\phi^{-1}\right)\left(h_{2}\right):=\phi^{-1} \circ h_{2} \circ \phi=h_{1}$. We denote by $I s o(\mathcal{F})$ the isotropy group of the germ of foliation $(\mathcal{F}: \omega=0)$ in $\left(\mathbb{C}^{2}, 0\right)$, defined by

$$
\operatorname{Iso}(\mathcal{F})=\left\{\phi \in \operatorname{Diff}\left(\mathbb{C}^{2}, 0\right): \phi^{*} \omega \wedge \omega=0\right\}
$$

Further, let us denote the Hopf bundle of Chern class $-k$ by $\mathbb{H}(-k):\left(p_{(k)}: \mathcal{H}(-k) \rightarrow \mathbb{C P}(1)\right)$, or just by its total space $\mathbb{H}(-k)$. Recall, from the theory of Algebraic Curves, that whenever $\pi:(\tilde{X}, D) \longrightarrow\left(\mathbb{C}^{2}, 0\right)$ is a map resulting from the iteration of finite number of blow-ups, with exceptional curve $D=\pi^{-1}(0)$, whose irreducible components are $D_{j}, j=1 \cdots n$, with Chern classes $-k_{j}$, then a suitable neighborhood of $D$ in $\widetilde{X}$ results from pasting together suitable neighborhoods of the zero sections of $\mathbb{H}\left(-k_{j}\right)$. Thus, for each Hopf bundle component $p: \mathcal{H}_{j} \rightarrow$ 
$D_{j}$ of a given resolution, we shall denote by $\widetilde{\mathcal{F}}_{j}$, the germ of foliation at $D_{j} \subset \mathcal{H}_{j}$ induced by the restriction of $\widetilde{\mathcal{F}}$, and call it the $j^{\text {th }}$ Hopf component of the resolution. Further, recall that $\widetilde{\mathcal{F}}_{j}$ is nondicritical if $D_{j}$ is an invariant set of the foliation, and dicritical otherwise. In the former case, the holonomy of $\widetilde{\mathcal{F}}_{j}$ with respect to a section $\Sigma$ transversal to $D \simeq \mathbb{C P}(1)$ is called the projective holonomy of $\widetilde{\mathcal{F}}_{j}$, and denoted by $\operatorname{Hol}_{\Sigma}\left(\mathcal{F}_{j}, D_{j}\right)$, furthermore one says that it is solved if it has just reduced singularities (cf. Mattei and Moussu 1980), and rectified if $\operatorname{Sep}\left(\widetilde{\mathcal{F}}_{j}\right)$ is contained in a fibration transversal to $D_{j}$ in $\left(\mathcal{H}_{j}, D_{j}\right)$. In both cases one calls the set of singularities of $\widetilde{\mathcal{F}}_{j}$ the first tangent cone of $\mathcal{F}_{j}$, and, in the later case, the set of tangencies between $\widetilde{\mathcal{F}}_{j}$ and $D_{j}$ the second tangent cone of $\widetilde{\mathcal{F}}$.

Let $\widetilde{\mathcal{F}}^{i}, i=1,2$, be two germs of singular nondicritical foliations at $\mathbb{C P}(1) \subset \mathcal{H}$, and $\varphi \in$ $P G L(2, \mathbb{C})$ be an isomorphism between their sets of singular points $\left\{t_{j}^{i}\right\}_{j=1}^{n}$, that is $\varphi\left(t_{j}^{1}\right)=t_{j}^{2}$. Further let $t_{0}^{1} \in \mathbb{C P}(1)$ be a regular point of $\widetilde{\mathcal{F}}^{1}, t_{0}^{2}=\varphi\left(t_{0}^{1}\right)$, and denote by $h_{\gamma}^{i}$ the holonomy of a path $\gamma \in$ $\pi_{1}\left(\mathbb{C P}(1) \backslash\left\{t_{j}^{i}\right\}_{j=1}^{n}, t_{0}^{i}\right)$, with respect to sections $\Sigma_{i}, i=1,2$ transversal to $\mathbb{C P}(1)$. Then recall that the projective holonomies of these foliations have an analytic conjugation subordinated to $\varphi$ if there is $\phi \in \operatorname{Diff}(\mathbb{C}, 0)$ such that $A d\left(\phi^{-1}\right)\left(h_{\gamma}^{2}\right)=h_{\varphi^{*} \gamma}^{1}$, for every $\gamma \in \pi_{1}\left(\mathbb{C P}(1) \backslash\left\{t_{j}^{1}\right\}_{j=1}^{n}, t_{0}^{1}\right)$. Further we set $\operatorname{Diff}\left(\widetilde{\mathcal{F}}^{1}, \widetilde{\mathcal{F}}^{2}\right)=\left\{\Phi \in \operatorname{Diff}(\mathcal{H}, \mathbb{C P}(1)): \Phi_{*}\left(\widetilde{\mathcal{F}}^{1}\right)=\widetilde{\mathcal{F}}^{2}, \Phi\left(\operatorname{Sing}\left(\widetilde{\mathcal{F}}^{1}\right)\right)=\varphi\left(\operatorname{Sing}\left(\widetilde{\mathcal{F}}^{1}\right)\right\}\right.$. In particular we denote the isotropy group of $\widetilde{\mathcal{F}}$ by $\operatorname{Iso}(\widetilde{\mathcal{F}})=\operatorname{Diff}(\widetilde{\mathcal{F}}, \widetilde{\mathcal{F}})$.

We say that a resolution of a nondicritical foliation is simple if its exceptional divisor has only one projective line with three or more singularities (see figure 1), which is called principal projective line of the exceptional divisor; its holonomy is called the projective holonomy of the foliation. In particular the Hopf component of a simple nondicritical foliation about the principal project line is called the principal Hopf component.

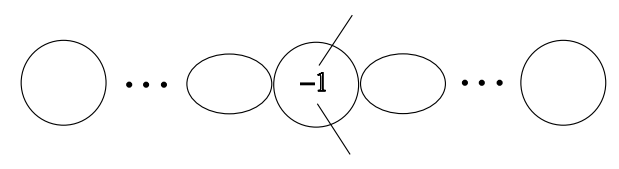

Fig. 1 - A simple resolution for a nondicritical foliation.

Now recall that a saddle-node is a singularity with a degenerated nonvanishing linear part which presents two invariant curves passing through the origin: the strong variety which is analytic and a formal one, which is called central variety when it converges. Their holonomies are called strong holonomy in the former case, and weak holonomy in the later. Let $\widetilde{\mathcal{F}}$ be a nondicritical solved foliation defined around the zero section of a Hopf bundle $\mathbb{H}:(p: \mathcal{H} \rightarrow D)$, with saddle-nodes along the divisor. Then one says that a saddle-node defined in $(\mathcal{H}, D)$ is transversal if its strong variety is transversal to $D$. Otherwise one say that the saddle-node is parallel.

Recall from Dulac 1904 that every saddle-node $(\mathcal{F}: \omega=0)$ can be analytically reduced to the form $\left(\mathcal{F}_{p, A}: \omega_{p, A}=0\right)$ where

$$
\omega_{p, A}(x, y)=x^{p+1} d y-A(x, y) d x
$$


with $A \in \mathcal{O}_{2}$ and $A(0, y)=y$. That is, there is $\Phi_{p, A} \in \operatorname{Diff}\left(\mathbb{C}^{2}, 0\right)$ such that

$$
\left(\Phi_{p, A}\right)^{*} \omega_{p, A} \wedge \omega=0
$$

which will be called an analytic Dulac reduction. On the other hand each singularity of the form $\left(\mathcal{F}_{p, A}: \omega_{p, A}=0\right)$ has a formal normal form $\left(\mathcal{F}_{p, \lambda}: \omega_{p, \lambda}=0\right)$ where

$$
\omega_{p, \lambda}(x, y)=x^{p+1} d y-y\left(1+\lambda x^{p}\right) d x
$$

In other words, there is $\widehat{N}\left(\mathcal{F}_{p, A}\right) \in \widehat{\operatorname{Diff}}_{\mathbf{I}}\left(\mathbb{C}^{2}, 0\right)$ given by $\widehat{N}\left(\mathcal{F}_{p, A}\right)(x, y)=(x, g(x, y))$, with $g(x, y)=y+\sum_{j \geq 1} a_{j}(y) x^{j}$, where all $a_{j}$ converge with the same radius of convergence, and such that

$$
\left(\widehat{N}\left(\mathcal{F}_{p, A}\right)\right)^{*} \omega_{p, \lambda} \wedge \omega_{p, A}=0,
$$

which will be called a formal Dulac reduction. Furthermore, for each $U_{j}=\left\{(x, y) \in \mathbb{C}^{2}: \arg (x)-\right.$ $\left.\frac{(2 j+1)}{2 p} \pi \in\right]-\pi / p, \pi / p[, 0<|y|<r, r \sim 0)$, with $j \in \mathbb{Z}_{2 p}$, there is an unique holomorphic transformation of $U_{j}$, namely $N_{j}\left(\mathcal{F}_{p, A}\right)$, such that $N_{j}\left(\mathcal{F}_{p, A}\right) \longrightarrow \widehat{N}\left(\mathcal{F}_{p, A}\right)$, i.e. $\widehat{N}\left(\mathcal{F}_{p, A}\right)$ is the asymptotic expansion of each $N_{j}(\mathcal{F})$ (in fact $p$-summable, cf. Hukuhara et al. 1961, Martinet and Ramis 1982) satisfying

$$
\left(N_{j}\left(\mathcal{F}_{p, A}\right)\right)^{*} \omega_{p, \lambda} \wedge \omega_{p, A}=0
$$

Now let $\widetilde{U}_{j}:=\Phi_{p, A}^{-1}\left(U_{j}\right)$ and $N_{j}(\mathcal{F}):=N_{j}\left(\mathcal{F}_{p, A}\right) \circ \Phi_{p, A}: \widetilde{U}_{j} \longrightarrow U_{j}$ for $j \in \mathbb{Z}_{2 p}$. Then one has that $N_{j+1, j}(\mathcal{F}):=N_{j+1}(\mathcal{F}) \circ N_{j}(\mathcal{F})^{-1}$ can be identified with $\mathbb{C}$ for $j=0$ mod 2 and with $\operatorname{Diff}_{\mathbf{I}}(\mathbb{C}, 0)$ for $j \equiv 1 \bmod 2$. Hence one has that (up to a linear change of coordinates $(x, y) \mapsto(\alpha x, \beta y)$, with $\alpha^{p}=1$, and $\left.\beta \in \mathbb{C}^{*}\right)$ the map

$$
\begin{array}{rlcc}
\Theta: & \Sigma_{p, \lambda} & \longrightarrow & \mathbb{C} \times \operatorname{Diff}_{\mathbf{I}}(\mathbb{C}, 0) \times \stackrel{p \text {-times }}{\cdots} \times \mathbb{C} \times \operatorname{Diff}_{\mathbf{I}}(\mathbb{C}, 0) \\
(\mathcal{F}: \omega=0) & \longmapsto & \left(N_{1,0}(\mathcal{F}), N_{2,1}(\mathcal{F}), \cdots, N_{2 p-1,2 p-2}(\mathcal{F}), N_{2 p, 2 p-1}(\mathcal{F})\right)
\end{array}
$$

is a well defined biholomorphism, where $\Sigma_{p, \lambda}$ is the orbit space under analytic equivalence of the set of saddle-nodes with formal normal form $\left(\mathcal{F}_{p, \lambda}: \omega_{p, \lambda}=0\right)$. In particular we have that $(\mathcal{F}: \omega=0)$ has central variety if and only if $\Theta(\mathcal{F})=\left(\mathbf{I}, N_{2,1}(\mathcal{F}), \cdots, \mathbf{I}, N_{2 p, 2 p-1}(\mathcal{F})\right)$. Note that to determine the analytic type of a saddle-node, it suffices to determine $N_{j}(\mathcal{F})$ for all $j \in \mathbb{Z}_{2 p}$ (Martinet and Ramis 1982). So we say that $\left(\widetilde{U}_{j}, N_{j}(\mathcal{F})\right)$ is a sectorial chart and that $\bigcup_{j \in \mathbb{Z}_{2 p}}\left(\widetilde{U}_{j}, N_{j}(\mathcal{F})\right)$ is a sectorial atlas for the saddle-node $(\mathcal{F}: \omega=0)$. Now let $\mathcal{F}^{1}, \mathcal{F}^{2} \in \Sigma_{p, \lambda}$ be two saddle-nodes with central variety and formal normal form $\mathcal{F}_{p, \lambda}$. Let $\Sigma_{a}=(x=a), \widetilde{\Sigma}_{a}^{j}:=\left(\Phi_{p, A}^{j}\right)^{-1}\left(\Sigma_{a}\right)$, $S_{j}:=\left(\Phi_{p, A}^{j}\right)^{-1}((y=0))$, and $\phi$ be a conjugation between $\operatorname{Hol}_{\widetilde{\Sigma}_{a}^{1}}\left(\mathcal{F}^{1}, S_{1}\right)$ and $\operatorname{Hol}_{\widetilde{\Sigma}_{a}^{2}}\left(\mathcal{F}^{2}, S_{2}\right)$. Then one says that $\phi$ is $p$-periodic in sectorial charts if

$$
\left.\left(\left.N_{j}\left(\mathcal{F}^{2}\right)\right|_{\widetilde{\Sigma}_{a}^{2}}\right) \circ \phi \circ\left(N_{j}\left(\mathcal{F}^{1}\right)\right)^{-1}\right|_{\Sigma_{a}}(y)=\exp \left(\frac{2 \pi i}{p}\right) \cdot y
$$

Henceforth, we say that a saddle-node with central variety has a $q$-symmetric Stokes phenomenon or has $q$-order of symmetry if $q$ is the greater integer for which the map $R_{q}(z)=\exp \left(\frac{2 \pi i}{q}\right) \cdot z$ 
commutes with $N_{j+1, j}$ for every $j \equiv 1 \bmod 2$. Let $\widetilde{\mathcal{F}}$ and $\widetilde{\mathcal{F}}^{\prime}$ be two analytically equivalent, up to order one (for reduced nondegenerate singularities) and formal type (for saddle-nodes), solved foliations defined in the Hopf bundle $\mathbb{H}:(p: \mathcal{H} \longrightarrow D)$, with $\left\{t_{j}\right\}_{j=1}^{n}$ and $\left\{t_{j}^{\prime}\right\}_{j=1}^{n}$ being transversal saddle-nodes, such that $t_{j}^{\prime}=\varphi\left(t_{j}\right)$, where $\varphi \in \operatorname{Diff}(D) \approx P G L(2, \mathbb{C})$ is their tree isomorphism, and with $\left.\widetilde{\mathcal{F}}\right|_{\left(D, t_{j}\right)}$ and $\widetilde{\mathcal{F}}_{\left.\right|_{\left(D, t_{j}\right)}}$ having the same order of symmetry, say $q_{j}$. Then one says that a holomorphic conjugation between $\operatorname{Hol}_{\Sigma}(\widetilde{\mathcal{F}}, D)$ and $\operatorname{Hol}_{\Sigma^{\prime}}\left(\widetilde{\mathcal{F}}^{\prime}, D\right)$ commutes with the stokes phenomenon of $\widetilde{\mathcal{F}}$ and $\widetilde{\mathcal{F}}^{\prime}$ if for each pair of equivalent points $\left(t_{j}, t_{j}^{\prime}\right), \phi$ is $p_{j}$-periodic in sectorial charts, where $p_{j}$ is a divisor of $\operatorname{gcd}\left(\left\{q_{j}\right\}_{j=1}^{n}\right)$. Let $\mathcal{F}$ be a singular foliation in $\left(\mathbb{C}^{2}, 0\right)$ with dicritical component, and $\widetilde{\mathcal{F}}$ its extended resolution, then one says that each nondicritical Hopf component that does not intercept a dicritical Hopf component is a nondicritical cell. Further, if $\mathcal{F}$ has a dicritical component, then the union of each dicritical Hopf component with the nondicritical ones intercepting it, is called a dicritical cell, and denoted by $\widetilde{\mathcal{C}}_{j}=\widetilde{\mathcal{F}}_{j, 0} \cup_{k=1}^{n} \widetilde{\mathcal{F}}_{j, k}$ where $\widetilde{\mathcal{F}}_{j, 0}$ is the dicritical component of the cell.
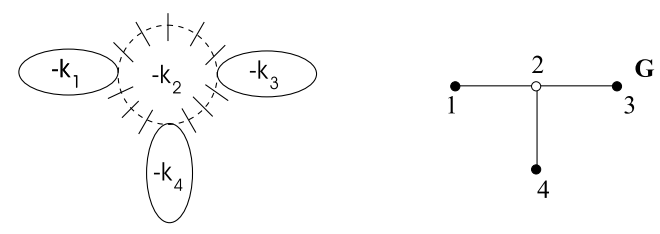

Fig. 2 -A dicritical cell.

Let $\widetilde{\mathcal{C}}^{i}=\widetilde{\mathcal{F}}_{0}^{i} \cup_{k=1}^{n} \widetilde{\mathcal{F}}_{k}^{i}, i=1,2$, be two germs of dicritical cells, where each $\widetilde{\mathcal{F}}_{k}^{i}$ is defined at the zero section of the Hopf bundle $\mathbb{H}_{k}:\left(p_{k}: \mathcal{H}_{k} \longrightarrow D_{k}\right)$. Then one says that $\prod_{k=1}^{n} \operatorname{Hol}_{D_{0}}\left(\widetilde{\mathcal{F}}_{k}^{1}, D_{k}\right)$ and $\prod_{k=1}^{n} \operatorname{Hol}_{D_{0}}\left(\widetilde{\mathcal{F}}_{k}^{2}, D_{k}\right)$ have a Möbius conjugation if there is $\phi \in \operatorname{Diff}\left(D_{0}\right) \simeq \operatorname{PGL}(2, \mathbb{C})$ such that $\operatorname{Ad}(\phi)\left(\operatorname{Hol}_{D_{0}}\left(\widetilde{\mathcal{F}}_{k}^{2}, D_{k}\right)\right)=\operatorname{Hol}_{D_{0}}\left(\widetilde{\mathcal{F}}_{k}^{1}, D_{k}\right)$. Furthermore, let $\varphi_{k} \in \operatorname{Diff}\left(D_{k}\right) \simeq P G L(2, \mathbb{C})$ be an isomorphism between $\operatorname{Sing}\left(\widetilde{\mathcal{F}}_{k}^{1}\right)$ and $\operatorname{Sing}\left(\widetilde{\mathcal{F}}_{k}^{2}\right)$ for $k=1 \cdots n$ and between the corners of $\widetilde{\mathcal{F}}_{0}^{1}$ and $\widetilde{\mathcal{F}}_{0}^{2}$. Then we say that a Möbius conjugation is subordinated to $\varphi:=\left(\varphi_{k}\right)_{k=0}^{n}$ if it is subordinated

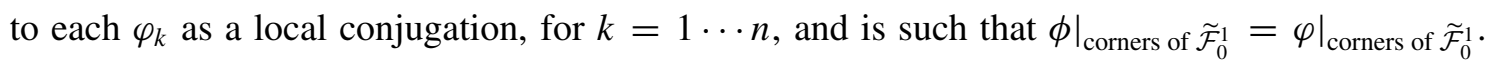
Further we set $\mathcal{B H}:=\mathcal{H}_{0} \cup_{k=1}^{n} \mathcal{H}_{k}$ and $B D:=D_{0} \cup_{k=1}^{n} D_{k}$ and define $\operatorname{Diff}(\mathcal{B H}, B D)$ to be the set of diffeomorphisms of $\mathcal{B H}$ preserving $D_{k}$, for $k=0 \cdots n$. Moreover we set $\operatorname{Diff}_{\widetilde{\mathcal{C}}^{1}, \widetilde{\mathcal{C}}^{2}}(\mathcal{B H}, B D):=$ $\left\{\Phi \in \operatorname{Diff}(\mathcal{B H}, B D): \Phi_{*}\left(\widetilde{\mathcal{C}}^{1}\right)=\widetilde{\mathcal{C}}^{2}, \Phi\left(\operatorname{Sing}\left(\widetilde{\mathcal{F}}_{k}^{1}\right)\right)=\varphi\left(\operatorname{Sing}\left(\widetilde{\mathcal{F}}_{k}^{1}\right)\right\}\right.$. In particular we define the isotropy group of the dicritical cell $\widetilde{\mathcal{C}}$ by $\operatorname{Iso}(\widetilde{\mathcal{C}}):=\operatorname{Diff}_{\widetilde{\mathcal{C}}, \widetilde{\mathcal{C}}}(\mathcal{B H}, B D)$. Also we shall say that a dicritical cell is solved if each of its singularities are reduced. Analogously we shall say that a dicritical cell is rectified if each of its nondicritical component is solved and rectified, and its dicritical component has void first and second tangent cones. Note that two dicritical cells may intersect, but if it happens, it most be at just one nondicritical Hopf component of the foliation. Let us call the larger simply-connected subsets of the resolution space, containing just nondicritical Hopf components of the foliation, the nondicritical branches of $\widetilde{\mathcal{F}}$. In particular, we shall say that a nondicritical branch is simple if it has just one projective line with three or more singularities, 
which shall be called the principal projective line of the branch. So we extend the concept of simple resolution foliation in the following way: we say that a resolution of a dicritical foliation is simple whenever its has just one dicritical component, and each nondicritical branch is simple with principal projective line intercepting the dicritical component (see figure 3).

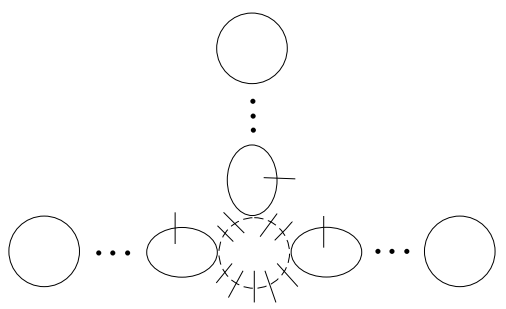

Fig. 3 - A dicritical foliation with simple extended rectifier resolution.

Further, let $\widetilde{\mathcal{F}}$ be a simple nondicritical branch of a solved and rectified foliation. Then we say that a dicritical singular foliation, say $\widetilde{\mathcal{G}}$, is a companion fibration for $\widetilde{\mathcal{F}}$, if $\widetilde{\mathcal{G}}$ satisfies the following properties:

(1) $\widetilde{\mathcal{F}}$ and $\widetilde{\mathcal{G}}$ have the same chain of projective lines (cf. Câmara 2001);

(2) $\widetilde{\mathcal{G}}$ is simple and its (only) dicritical component is a fibration transversal (cf. Câmara 2001) to the principal Hopf component of $\widetilde{\mathcal{F}}$;

We say that a singular foliation $(\mathcal{F}: \omega=0)$ has an (extended) rectifier resolution, say $(\widetilde{\mathcal{F}}: \widetilde{\omega}=0)$ if each of its Hopf components, namely $\widetilde{\mathcal{F}}_{j}$, satisfies one of the following conditions:

(1) $\widetilde{\mathcal{F}}_{j}$ is nondicritical, solved, rectified and does not have parallel saddle-nodes;

(2) $\widetilde{\mathcal{F}}_{j}$ has just two singularities, and in antipodal points of $D_{j} \approx \mathbb{C P}(1)$ : a parallel saddle-node and a (corner) resonant saddle with index -1 with respect to $D_{j}$;

(3) $\widetilde{\mathcal{F}}_{j}$ is dicritical and has void first and second tangent cones.

Notice that, similarly to the case of Seidenberg's resolution, we can talk about a minimal rectifier resolution, although this is not unique in general. Two rectifier resolutions, namely $\widetilde{\mathcal{F}}^{1}$ and $\tilde{\mathcal{F}}^{2}$, are cell-wise equivalent if they have isomorphic trees of singularities and there is a collection of biholomorphisms $\Phi_{j}$ taking the leaves of $\widetilde{\mathcal{C}}_{j}{ }^{1}$ into the leaves of $\widetilde{\mathcal{C}}_{j}{ }^{2}$. In particular, we denote by $\sum_{\omega}^{\mathrm{c}}$ (respect. $\sum_{\omega}^{\mathrm{c}, 1, \mathrm{~F}}$ ) the set of germs of foliations which has a minimal (extended) rectifier resolution cell-wise equivalent (respect. up to order one for the nondegenerate reduced singularities and formal type for the saddle-nodes) to a minimal rectifier resolution of $(\mathcal{F}: \omega=0)$. Further we denote as $\sum_{\omega, f}^{\mathrm{c}, 1, \mathrm{~F}}$ the subset of $\sum_{\omega}^{\mathrm{c}, 1, \mathrm{~F}}$ whose separatrix set has the same analytic type of the curve $f^{-1}(0)$. 


\section{STATEMENT OF THE MAIN RESULTS}

The above definitions of rectifier resolution are supported by the following.

Proposition 1. Every germ of analytic foliation in $\left(\mathbb{C}^{2}, 0\right)$ has an (extended) rectifier resolution.

Let $(\mathcal{F}: \omega=0)$ be a germ of foliation in $\left(\mathbb{C}^{2}, 0\right)$, then pick a foliation $\mathcal{F}^{\text {o }}$ cell-wise isomorphic to $\mathcal{F}$, which shall be called a fixed model for $\mathcal{F}$, and consider the elements $\Phi_{j} \in \operatorname{Diff}\left(\widetilde{\mathcal{C}_{j}}, \widetilde{\mathcal{C}_{j}^{\text {o }}}\right)$, which shall be called cell charts for the $j^{\text {th }}$ cell of the fixed model. Then it is straightforward that for each cell $\widetilde{\mathcal{C}_{j}}$ and each fixed model cell $\widetilde{\mathcal{C}_{j}^{o}}$, there exists only one cell chart, up to left composition by an element of $I \operatorname{so}\left(\widetilde{\mathcal{C}}_{j}^{\mathrm{o}}\right)$. So, consider the sheaf of nonabelian groups $\Lambda^{\mathrm{o}}:=\operatorname{Iso}\left(\widetilde{\mathcal{F}}^{\mathrm{o}}\right)$, then we say that $\mathcal{U}:=\cup U_{j}$ is a good covering for $\Lambda^{\circ}$ if $U_{j}$ are neighborhoods of $D_{j} \subset \mathcal{H}_{j}$ or of $B D_{j} \subset \mathcal{B H}_{j}$, respectively for nondicritical or dicritical cells. Therefore, consider the first cohomology set $H^{1}\left(\mathcal{U}, \Lambda^{\circ}\right)$ associated to the good covering $\mathcal{U}$, and set $H^{1}\left(D, \Lambda^{\circ}\right)$ as the direct limit of $H^{1}\left(\mathcal{U}, \Lambda^{\circ}\right)$ for the good coverings for of $\Lambda^{\circ}$, associated to $D=\cup D_{j}$, the exceptional divisor of a minimal rectifier resolution of $\mathcal{F}$.

Thus we define the map

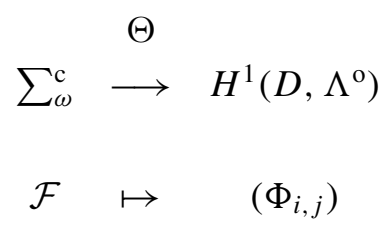

where $\Phi_{i, j}:=\Phi_{i} \circ \Phi_{j}^{-1}$. Note that $\Theta$ does not depend on the fixed models up to cell-wise conjugacy class. Then, as a consequence of path-lifting construction, one has that:

THEOREM 1. Let $\mathcal{F}, \mathcal{F}^{\prime}$ be two germs of singular foliations in $\left(\mathbb{C}^{2}, 0\right)$, and $\widetilde{\mathcal{F}}, \widetilde{\mathcal{F}}^{\prime}$ one of their minimal rectifier resolutions respectively. Then $\mathcal{F}, \mathcal{F}^{\prime}$ are analytically equivalent if and only if they satisfy:

(1) $\mathcal{F}, \mathcal{F}^{\prime} \in \sum_{\omega}^{\mathrm{c}, 1, \mathrm{~F}}$ for some 1 -form $\omega \in \Omega^{1}\left(\mathbb{C}^{2}, 0\right)$ (holomorphic one-forms). In particular we denote by $\varphi=\left(\varphi_{j}\right): \Gamma_{s} \longrightarrow \Gamma_{s}^{\prime}$ the component-wise isomorphism between the trees of singularities of their minimal rectifier resolution.

(2) Two equivalent (up to formal type) transversal saddle-nodes $t_{i, j}, t_{i, j}^{\prime}=\varphi_{j}\left(t_{i, j}\right)$ have the same order of symmetry.

(3) For each nondicritical cell $\widetilde{\mathcal{F}}_{j}$ and $\widetilde{\mathcal{F}}_{j}^{\prime}$ their projective holonomies have an analytic conjugation, namely $\phi_{j}$, subordinated to $\varphi_{j}$, and commuting with the Stokes phenomenon of the transversal saddle-nodes of $\widetilde{\mathcal{F}}_{j}$ and $\widetilde{\mathcal{F}}_{j}^{\prime}$.

(4) For each dicritical cell $\widetilde{\mathcal{C}}_{j}=\widetilde{\mathcal{F}}_{j, 0} \cup_{k=1}^{n_{j}} \widetilde{\mathcal{F}}_{j, k}$ and $\widetilde{\mathcal{C}}_{j}^{\prime}=\widetilde{\mathcal{F}}_{j, 0}^{\prime} \cup_{k=1}^{n_{j}} \widetilde{\mathcal{F}}_{j, k}^{\prime}$, we have that $\prod_{k=1}^{n_{j}} \operatorname{Hol}_{D_{j, 0}}\left(\widetilde{\mathcal{F}}_{j, k}, D_{j, k}\right)$ and $\prod_{k=1}^{n_{j}} \operatorname{Hol}_{D_{j, 0}}\left(\widetilde{\mathcal{F}}_{j, k}^{\prime}, D_{j, k}\right)$ have a Möbius conjugation, namely $\phi_{j}$, subordinated to $\varphi$, and commuting with the Stokes phenomenon of the transversal saddlenodes of $\widetilde{\mathcal{F}}_{j, k}$ and $\tilde{\mathcal{F}}_{j, k}^{\prime}$. 
(5) $\Theta(\mathcal{F})=\Theta\left(\mathcal{F}^{\prime}\right)$, for one (and every) fixed model of $\sum_{\omega}^{c}$.

Notice that (2) above answers in affirmative way the following conjecture of S. M. Voronin (cf. Elizarov et al. 1993): Beyond the resolution tree of the minimal resolution and the projective holonomies there are some further numerical invariants for singular foliations in $\left(\mathbb{C}^{2}, 0\right)$. In fact, these new invariants are the order of symmetry of each transversal saddle-node.

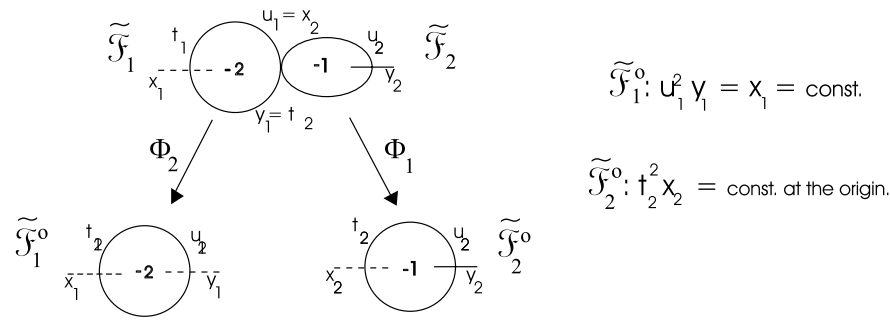

Fig. 4 - A fixed model for $\sum_{d\left(y^{2}+x^{4}\right)}^{c, 1}$.

Recall that in Câmara 2001, for $f(x, y)=\prod_{j=1}^{d}\left(y^{p}-\lambda_{j} x^{q}\right)$, with $1<p<q \in N^{*}$, g.c.d. $(p, q)=1$, and $\lambda_{j} \in \mathbb{C}^{*}$, we classify the set $\sum_{\omega, f}^{c, 1}$ (the subset of generalized curves of $\left.\sum_{\omega, f}^{\mathrm{c}, 1, \mathrm{~F}}\right)$ by its projective holonomy, generalizing a result of Cerveau and Moussu 1988 concerning the subset $\sum_{\omega, y^{2}+x^{n}}^{c, 1}$. Here we give a slight generalization of this result, including in particular a result of Meziani 1996 concerning $\sum_{\omega, y^{2}+x^{n}}^{\mathrm{c}, 1, \mathrm{~F}}$.

THeOREM 2. Let $f(x, y)=\prod_{j=1}^{d}\left(y^{p}-\lambda_{j} x^{q}\right)$, where $1<p<q \in N^{*}$, g.c.d. $(p, q)=1$, and $\lambda_{j} \in \mathbb{C}^{*}$. Then, for every $\mathcal{F} \in \sum_{\omega}^{c, 1, F}$ we have that $\mathcal{F}$ has a simple minimal rectifier resolution, and $\Theta(\mathcal{F})$ is trivial in $H^{1}\left(D, \Lambda^{o}\right)$. In particular if $\widetilde{\mathcal{F}}$ and $\widetilde{\mathcal{F}}^{\prime}$ are minimal rectifier resolutions of $\mathcal{F}, \mathcal{F}^{\prime} \in \sum_{\omega}^{c, 1, F}$, and $\widetilde{\mathcal{F}}_{0}, \widetilde{\mathcal{F}}_{0}^{\prime}$ their principal projective lines, respectively, then $\mathcal{F}$ and $\mathcal{F}^{\prime}$ are analytically equivalent if and only if:

(1) If $\varphi$ is the isomorphism between the trees of $\widetilde{\mathcal{F}}$ and $\widetilde{\mathcal{F}}^{\prime}$ with $t_{i j}^{\prime}=\varphi\left(t_{i j}\right)$, then $t_{i j}$ and $t_{i j}^{\prime}$ have the same order of symmetry, whenever they are transversal saddle-nodes;

(2) Their projective holonomies have a conjugation subordinated to $\varphi$ and commuting with the Stokes phenomenon of the transversal saddle-nodes of $\widetilde{\mathcal{F}}_{0}$ and $\widetilde{\mathcal{F}}_{0}^{\prime}$.

We would like to stress the importance of the fixed separatrix type in the above theorem, remarking that in general $\sum_{\omega, f}^{\mathrm{c}, 1, \mathrm{~F}} \varsubsetneqq \sum_{\omega}^{\mathrm{c}, 1, \mathrm{~F}}$.

THEOREM 3. Let $\mathcal{F}$ be a germ of dicritical foliation which has a simple minimal rectifier resolution, say $\widetilde{\mathcal{F}}$, whose nondicritical branches have a companion fibration. Then $\Theta(\mathcal{F})$ is trivial in $H^{1}\left(D, \Lambda^{o}\right)$. In particular if $\mathcal{F}, \mathcal{F}^{\prime} \in \sum_{\omega}^{c, 1, F}$ then they are analytically equivalent if and only if they satisfy: 
(1) If $\varphi$ is the isomorphism between the trees of $\widetilde{\mathcal{F}}$ and $\widetilde{\mathcal{F}}^{\prime}$ with $t_{i j}^{\prime}=\varphi\left(t_{i j}\right)$, then $\left.\widetilde{\mathcal{F}}\right|_{\left(D_{i}, t_{i j}\right)}$ and $\left.\widetilde{\mathcal{F}}^{\prime}\right|_{\left(D_{i}, t_{i j}^{\prime}\right)}$ have the same order of symmetry, whenever $t_{i j}$ and $t_{i j}^{\prime}$ are transversal saddle-nodes;

(2) For any dicritical cells $\widetilde{\mathcal{C}}_{j}=\widetilde{\mathcal{F}}_{j, 0} \cup_{k=1}^{n} \widetilde{\mathcal{F}}_{j, k}$ and $\widetilde{\mathcal{C}}_{j}^{\prime}=\widetilde{\mathcal{F}}_{j, 0}^{\prime} \cup_{k=1}^{n} \widetilde{\mathcal{F}}_{j, k}^{\prime}$, we have that $\prod_{k=1}^{n} \operatorname{Hol}_{D_{j, 0}}\left(\widetilde{\mathcal{F}}_{j, k}, D_{j, k}\right)$ and $\prod_{k=1}^{n} \operatorname{Hol}_{D_{j, 0}}\left(\widetilde{\mathcal{F}}_{j, k}^{\prime}, D_{j, k}\right)$ have a Möbius conjugation, namely $\phi$, subordinated to $\varphi$, and commuting with the Stokes phenomenon of the transversal saddlenodes of $\widetilde{\mathcal{F}}_{j, k}$ and $\tilde{\mathcal{F}}_{j, k}^{\prime}$.

A particular instance of a foliation in the hypothesis of the above theorem happens when the minimal (Seidenberg) resolution of a dicritical singularity is simple and has just tangencies and the kind of singularities we study in theorem 2, in the dicritical component, generalizing some results of Klughertz 1988. We would like to remark that the stated theorems are proved in Câmara 2003. Furthermore, in a forthcoming work we shall describe more precisely $H^{1}\left(D, \Lambda^{0}\right)$ showing that, roughly speaking, it can be split in the first cohomology group of the coherent sheaf of vector fields tangent to the model $\mathcal{F}^{\mathrm{o}}$ and the first cohomology group of a (in general finitely generated) sheaf of abelian groups generated by the centralizers of the projective holonomy groups of $\widetilde{\mathcal{F}}_{j}^{o}$. In particular we will present some examples for which the cohomology is nontrivial.

\section{RESUMO}

Estudamos a classificação de equações diferenciais analíticas em $\left(\mathbb{C}^{2}, 0\right)$ fornecendo uma lista completa de invariantes analíticos que determinam o tipo analítico da folheação subjacente. Em particular respondemos afirmativamente a uma conjectura de S. Voronin e generalizamos de forma imediata alguns resultados preliminares a respeito de singularidades dicríticas. Tal problema tem suas orígens numa conjectura proposta por R. Thom em meados da década de 1970.

Palavras-chave: Folheações singulares, resolução de singularidades, holonomia, cohomologia nãoabeliana.

\section{REFERENCES}

Camacho C, Lins-Neto A And SAd P. 1984. Topological invariants and equidesingularization for holomorphic vector fields. J Diff Geom 20: 143-174.

CÂMara LM. 2001. Non-linear differential equations and their analytic invariants. Preprint Serie C 2001/5 IMPA.

CÂMara LM. 2003. On the classification of analytic differential equations in $\left(\mathbb{C}^{2}, 0\right)$. Preprint Serie A 2003/224 IMPA.

Cerveau D. 1999. Feuilletages holomorphes de codimension 1. Reduction des singularités en petites dimensions et applications. Dynamique et Géometrie Complexes, Panor \& Syntheses. Soc Math France 8. 
Cerveau D and Moussu R. 1988. Groupes d'automorphismes de ( $\mathbb{C}, 0)$ et équations différentielles $y d y+\cdots=0$. Bull Soc Math France 116: 459-488.

Dulac H. 1904. Recherches sur les points singuliers des équations différentielles. Journal de l'Ecole Polytechnique 9(2): 1-125.

Elizarov PM, IL'Yashenko YuS, Sherbakov AA and Voronin SM. 1993. Finitely generated groups of germs of one-dimensional conformal mappings, and invariants for complex singular points of analytic foliations of the complex plane. Collection: Nonlinear Stokes phenomena. Adv Soviet Math 14: $57-105$.

Hukuhara H, Kimura T and Matuda T. 1961. Équations différentielles ordinaires du premier ordre dans le champ complexe. Publ Math Soc of Japan 7.

KLughertz M. 1988. Feuilletages holomorphes à singularité isolée ayant une infinité de courbes integrales. Thèse, Univ Toulouse III.

Martinet J And Ramis J-P. 1982. Problèmes de modules pour des équations différentielles nonlinéaires du premier ordre. Publ Math IHES 55: 63-164.

Martinet J AND Ramis J-P. 1983. Classification analytique des équations différentielles nonlinéaires résonnantes du premier ordre. Ann Sci Ecole Norm Sup 16(4): 571-621.

Mattei J-F and Moussu R. 1980. Holonomie et intégrales premières. Ann Sci Ecole Norm Sup 13(4): 469-523.

MeZIANi R. 1996. Classification analytique d'équations différentielles $y d y+\cdots=0$ et espace de modules. Bol Soc Brasil Mat 27: 23-53.

SeIDENBerg A. 1968. Reduction of the singularities of the differential equation $A d y=B d x$. Amer J Math 90: 248-269. 